\title{
The Investigation of Multiple Intelligence Modalities of University Students Receiving Sports Education
}

\section{Mevlüt Yildiz ${ }^{1}$ \\ Yavuz Öntürk $^{2}$ (1) \\ Engin Efek $^{3}$ (D)}

'Faculty of Sport Sciences, Mugla Sitki Kocman University, Mugla, Turkey.

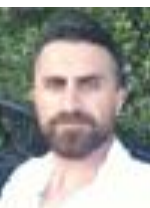

Corresponding Author)

Email:mevlutyildiz@mu.edu.trTel:+902522113171

${ }^{2}$ Faculty of Sport Sciences, $T$ alova University, Talova, Turkey.

Email:yavuzonturh@hotmail.com Tel: +902268155000

${ }^{s}$ Faculty of Sport Sciences, Duzce University, Duzce, Turkey.

Email:enginefel@hotmail.com Tel: +90 3805421215

\section{Abstract}

The aim of this study is to determine the dominant intelligence modality of university students who receive sports education and to examine intelligence modality in terms of various variables. The survey model was chosen for the model of the study. 251 students voluntarily participated in the study. A 6-question Personal Information Form was used by the researchers to determine the demographic characteristics of the participants, and the Multiple Intelligence Scale adapted in Turkish by Babacan and Dilci (2012) was used to determine the multiple intelligence areas. According to the findings, the intelligence modality in which the participants were the most dominant was physical intelligence, and the intelligence modality in which they were the most distant was the verbal intelligence. While multiple intelligence areas differed according to the gender, class, age and sports time of the participants, there was no significant difference in multiple intelligence areas according to department and branch variables. In addition, female participants, 3rd grade students and those who have more time to do sports are more dominant in every intelligence field. As a result, it was concluded that the most dominant intelligence field is physical intelligence and that women are more dominant in multiple intelligence domains than men. It was once again revealed that the duration of sports is an important factor in the development of multiple intelligence areas of individuals.

Keywords: Sport, Multiple intelligence, University students, Physical education, Education.

Citation | Mevlüt Yildiz; Yavuz Öntürk; Engin Efek (2020). The Investigation of Multiple Intelligence Modalities of University Students Receiving Sports Education. Asian Journal of Education and Training, 6(2): 246-255.

History:

Received: 16 January 2020

Revised: 3 March 2020

Accepted: 6 April 2020

Published: 20 April 2020

Licensed: This work is licensed under a Creative Commons

Attribution 3.0 License $(\boldsymbol{c c})$ E

Publisher: Asian Online Journal Publishing Group
Acknowledgement: Both authors contributed to the conception and design of the study.

Funding: This study received no specific financial support.

Competing Interests: The authors declare that they have no conflict of interests.

Transparency: The authors confirm that the manuscript is an honest, accurate, and transparent account of the study was reported; that no vital features of the study have been omitted; and that any discrepancies from the study as planned have been explained.

Ethical: This study follows all ethical practices during writing.

\section{Contents}

1. Introduction

2. Method

4. Discussion and Conclusion

References. 


\section{Contribution of this paper to the literature}

The study contributes to the existing literature by determining the most distant and dominant intelligence field of university students studying sports and also examines intelligence modality in terms of various variables.

\section{Introduction}

Although the concepts of learning and thinking do not mean the same, they are known as an inseparable whole. Confucius has an opinion on this matter: "Learning without an act of thinking ends with confusion." One of the objectives of learning is to provide the act of thinking. Due to individual differences, there are differences between individuals in thinking and multiple intelligence areas (Babacan \& Dilci, 2012; Chongde \& Tsingan, 2003). When the focus of learning is considered as an individual, it is important to know the strengths and weaknesses of the student, their past lives, choices, experiences and goals. Instead of allowing students to form indestructible taboos, it should be ensured that their educational decisions are directed towards their needs and expectations in their daily lives (Babacan \& Dilci, 2012; Gardner, 1999). In most of the intelligence theories, while people's grades and scores are used as criteria, it is seen as more important how, in which environment and when the individual learns in multiple intelligence theory (Babacan \& Dilci, 2012; Gardner, 1999). In this context, it is accepted as a serious mistake that individuals are measured only according to the scores, they get from IQ tests and that they are graded according to these measurement results. On the contrary, intelligence should not be seen as a phenomenon alone, it should be considered as a whole with different abilities (Armstrong, 2003; Babacan \& Dilci, 2012). Therefore, intelligence is a reflection of the structure of the brain, which consists of different parts (Babacan \& Dilci, 2012; Gardner, 1999). In his book, The Frames of the Mind, published in 1983, Gardner first collected intelligence types in seven areas. These types of intelligence are verbal, logical, musical, physical, visual, interpersonal, physical and internal intelligence. In 1995, an intelligence field was added to the seven intelligence fields advanced by Gardner. These 8 types of intelligence are: 1- Verbal - Linguistic Intelligence, 2- Logic - Mathematical Intelligence, 3Visual (Figure) - Spatial Intelligence, 4- Musical-Rhythmic and Harmonic Intelligence, 5- Bodily - Kinesthetic Intelligence, 6- Interpersonal - Social Intelligence, 7- Intrapersonal (Inner; Self-directed) Intelligence, 8Naturalistic Intelligence (İlhan, Mirzeoğlu, Aktaş, \& Demir, 2005). Although there are 8 kinds of intelligence types agreed upon, as the 9th intelligence type, Gardner emphasizes that Existentialist Intelligence is also a possible type of intelligence.

Identifying and knowing multiple intelligence areas of individuals is also an educational factor (Babacan \& Dilci, 2012). Identifying multiple intelligence trends, meeting the needs of students, preparing a curriculum suitable for learning styles, and knowing and implementing teaching strategies to enrich the learning experience of individuals are useful educational tools for both students and teachers (Armstrong, 2009; Babacan \& Dilci, 2012; Temız \& Kıraz, 2007). It is important for the person to know his intelligence characteristics well to adapt to his environment and achieve success in this existing environment (Başaran, 2004). For this reason, various education programs should be developed in the schools where the students are trained to experience their mental abilities and students should be actively involved in the educational process through teaching methods.

When the literature is analyzed, it can be seen that multiple intelligence theory is an important educational factor. For this reason, we think that the study will make an important contribution to the literature. On the other hand, studies on individuals or athletes who have received sports education are very few compared to other fields. In addition, in the majority of studies available in the literature (Baba, Karakaş, \& Gizdem, 2015; Ermiş, Ermiş, \& İmamoğlu, 2018; Kul, Bozkuş, Erol, \& Elçi, 2014; Metan \& Küçük, 2017; Oral, 2001; Tunç, 2008; Yenilmez \& Çalışkan, 2011) based on Gardner's previous studies, scales containing 7 or 8 intelligence areas were used. In this research, an up-to-date scale including 9 intelligence areas was used, and it was aimed to provide more up-to-date information to the literature in determining the multiple intelligence areas of its students.

Therefore, this factor expresses another importance of the research. Considering the current literature, in the studies of Bayrak, Celiksoy, and Celiksoy (2005); Hoşgörür and Katrancı (2007) and Kul et al. (2014) the field of intelligence that students of physical education and sports school were the most dominant is bodily / kinesthetic intelligence and verbal intelligence was the area of intelligence they were most distant from.

In the study of Ermiş et al. (2018) it was stated that the lowest intelligence field in adolescents who do sports and do not do sports is verbal intelligence. In addition, it was concluded that students doing sports during adulthood had positive effects on multiple intelligence scores. In the study carried out by Ürgüp (2015) and examining the multiple intelligence of physical education and sports school students studying in different departments, the closest intelligence field of the students was found as internal intelligence. In addition, the existential intelligence of the coaching students is more dominant than the other departments. In the study of Ermiş, İmamoğlu, and Erilli (2012) it was concluded that the sport positively affects the physical and social intelligence score and there is no difference in physical and social intelligence scores among athletes. In the study of Güllü and Tekin (2009) it was reported that there was a significant difference according to the gender and class of the students. In addition, the verbal, logical, visual and internal intelligence of general high school students is better than that of sports high school students; it has been found that only physical intelligence of sports high school students is better than general high school students. In the study of Metan and Küçük (2017) although there is no significant difference in most of the intelligence areas, significant results were found in favor of those who do not exercise regularly in some areas and in others.

In the study of Aslan, Dalkıran, and Ozer (2015) it was revealed that there are differences in the dominant intelligence areas of the athletes who practice the "periodic and aperiodic" sports branches. In the study of Kul et al. (2014) significant differences were observed in the different dimensions of the multiple intelligence areas of the candidates who participated in physical education and sports school examinations and those who could not win. In the study of Baba et al. (2015) while there was no significant differences according to gender, grade, and sport branch in the bodily/kinesthetic intelligence levels of the students in the school of physical education and sports, a significant relationship was observed between bodily/kinesthetic intelligence and academic achievement. 
In this context, the aim of this study was to determine the dominant intelligence modality of university students who receive sports education and to examine intelligence modality in terms of various variables (gender, grade, department, age, sport age, and branch). In the study, it was assumed that the participants answered the scale questions sincerely, the research sample represented the population, and the scale used in the research was able to measure the multiple intelligence modalities of the students. In addition, the research was limited to the scale used and to students studying at the Faculty of Sports Sciences at Duzce University in the 2019-2020 academic years.

The hypotheses of the research are listed as follows:

1. While the most dominant intelligence field of students studying in the field of sports sciences is physical / kinesthetic intelligence, the most distant intelligence field is verbal intelligence.

2. There are significant differences in multiple intelligence areas according to the students' gender.

3. There are significant differences in multiple intelligence areas according to students' grade levels.

4. There are significant differences in multiple intelligence areas according to the students' departments.

5. There are significant differences in multiple intelligence areas according to the students' ages.

6. There are significant differences in the areas of multiple intelligence according to the duration of the students' sports.

7. There are significant differences in multiple intelligence areas according to the branches that students make.

\section{Method}

2.1. Research model

The survey model, which is quantitative, was chosen for the model of the study.

\subsection{Study Group}

The population of the research was students who were studying at the Faculty of Sports Sciences at Duzce University in the 2019-2020 academic year, and the sample was randomly chosen 251 students who voluntarily participated in the research. Scale application study was carried out by the researchers in the classroom.

\subsection{Data Collection Instruments}

A 6-question Personal Information Form was used by the researchers to determine the demographic characteristics of the participants. the Multiple Intelligence Scale, developed by McClellan and Conti (2008) adapted to Turkish by Babacan and Dilci (2012) was used to determine the multiple intelligence areas. The scale consists of 3 sections and 27 items in total, 9 items in each section. Participants were asked to make a correct order from the expression they see closest to them to the expression they see as the most distant. The participants place 1 point next to the expression they feel closest to, and 9 points next to the expression they think is the most distant. Which intelligence field the participants are most prone to is determined by specifying the lowest total score they give to the items representing the intelligence field. The rankings of the questions in each intelligence area are summed up and the intelligence area with the lowest score is considered to be the intelligence area where the respondents predominate. The lowest score obtained from the scale is 3, and the highest score is 27 . The internal consistency coefficient (Cronbach's alpha coefficient) of the scale was found to be 0.90 .

\subsection{Data Analysis}

The data were analyzed using the SPSS 17 program. Frequency, percentage, minimum, maximum, average and standard deviation values were used in the analysis of the data. Shapiro Wilk-W test and Skewness-Kurtosis values were evaluated for normal distribution, t-test and ANOVA test were used since the data showed normal distribution, and Tukey test was used for Post-Hoc tests. Significance level was determined as p <0.05.

\section{Results}

In this part of the research, frequency, percentage, minimum, maximum, average and standard deviation values of the participants forming the research group were displayed. In addition, T-test, ANOVA test and Post-Hoc comparison results were presented in tables to determine whether the scores obtained from the scale differed according to gender, class, department, age, sports age and branch type.

When Table 1 is examined, it is seen that the majority of the participants are male (61.8\%). Likewise, it is seen that the majority of them attend fresher (33.5\%) and are students of the Department of Coaching education (45\%). Most of them are between $19-22$ years old $(70.5 \%)$ and sports age is 6-10 years old $(60.2 \%)$. In addition, the vast majority of the participants are engaged in team sports (53.8\%).

Sub-problem 1: Which intelligence areas are the most distant and dominant students in sports science education?

In Table 2, descriptive statistics on the multiple intelligence areas of students studying in the field of sports science are presented. The participants gave 1 point to the expression they felt closest to, and 9 points to the expression they felt distant to. When the scores are summed, the lowest scores represent the dominant intelligence field, and the highest scores represent the distant intelligence field (Babacan \& Dilci, 2012). Accordingly, the intelligence area which students are the most dominant is the physical intelligence area with an average of 8.51 . The intelligence area which the students are most distant is the verbal intelligence area with an average of 17.00.

Sub-problem 2: Do students' genders and intelligence areas differ significantly?

When Table 3 is analyzed, there were significant differences between genders regarding interpersonal/social, intrapersonal, logical/mathematical, Musical-Rhythmic/Harmonic, naturalistic, verbal and visual intelligence areas $(\mathrm{p}<0.05)$. There were no significant differences between the genders in bodily/kinesthetic and existential intelligence areas $(p>0.05)$. In addition, women were more dominant than men in all modalities.

Sub-problem 3: Do the intelligence areas of the students differ significantly according to their grades? 


\begin{tabular}{|c|c|c|c|}
\hline Variable & & $\mathbf{F}$ & $\%$ \\
\hline Gender & $\begin{array}{l}\text { Female } \\
\text { Male }\end{array}$ & $\begin{array}{c}96 \\
155 \\
\end{array}$ & $\begin{array}{l}38.2 \\
61,8 \\
\end{array}$ \\
\hline Grade & $\begin{array}{l}\text { Fresher } \\
\text { Sophomore } \\
\text { Third year } \\
\text { Final year }\end{array}$ & $\begin{array}{l}84 \\
26 \\
80 \\
61\end{array}$ & $\begin{array}{l}33,5 \\
10,4 \\
31,8 \\
24,3\end{array}$ \\
\hline Department & $\begin{array}{l}\text { Coaching Education } \\
\text { Physical Education and Sports Teaching } \\
\text { Sport Management }\end{array}$ & $\begin{array}{c}113 \\
96 \\
42\end{array}$ & $\begin{array}{l}45,0 \\
38,2 \\
16,8\end{array}$ \\
\hline Age & $\begin{array}{l}18 \text { years and under } \\
\text { Between } 19 \text { and } 22 \text { years } \\
\text { Between } 23 \text { and } 26 \text { years } \\
\text { Between } 27 \text { and } 29 \text { years } \\
30 \text { years and above }\end{array}$ & $\begin{array}{c}32 \\
177 \\
34 \\
1 \\
7 \\
\end{array}$ & $\begin{array}{c}12,8 \\
70,5 \\
13,5 \\
, 4 \\
2,8 \\
\end{array}$ \\
\hline Sport Age & $\begin{array}{l}5 \text { years and under } \\
\text { Between } 6-10 \text { years } \\
\text { Between } 11-15 \text { years } \\
16 \text { years and above }\end{array}$ & $\begin{array}{c}52 \\
151 \\
28 \\
20 \\
\end{array}$ & $\begin{array}{c}20,6 \\
60,2 \\
11,2 \\
8,0 \\
\end{array}$ \\
\hline Branch & $\begin{array}{l}\text { Team } \\
\text { Individual }\end{array}$ & $\begin{array}{l}135 \\
116 \\
\end{array}$ & $\begin{array}{l}53,8 \\
46,2 \\
\end{array}$ \\
\hline Total & & 251 & $100 \%$ \\
\hline
\end{tabular}

Table-2. Descriptive statistics for the multiple intelligence areas of the participants.

\begin{tabular}{l|c|c|c|c|c}
\hline Modality & $\mathbf{n}$ & Minimum & Maximum & Mean & S.D. \\
\hline Bodily/Kinesthetic & 251 & 3,00 & 24,00 & 8,51 & 5,11 \\
\hline Existential & 251 & 3,00 & 26,00 & 9,27 & 5,01 \\
\hline Interpersonal/Social & 251 & 3,00 & 27,00 & 10,32 & 5,34 \\
\hline Intrapersonal & 251 & 3,00 & 27,00 & 8,64 & 5,08 \\
\hline Logical/Mathematical & 251 & 3,00 & 27,00 & 8,96 & 5,39 \\
\hline Musical-Rhythmic/Harmonic & 251 & 3,00 & 27,00 & 13,38 & 6,13 \\
\hline Naturalistic & 251 & 3,00 & 25,00 & 10,92 & 6,19 \\
\hline Verbal/Linguistic & 251 & 3,00 & 27,00 & 17,00 & 6,25 \\
\hline Visual/Spatial & 251 & 3,00 & 27,00 & 13,78 & \\
\hline
\end{tabular}

Note: $\mathrm{p}<0.05$

Table-3. Differences between genders in terms of multiple intelligence.

\begin{tabular}{|c|c|c|c|c|c|c|c|}
\hline Modality & Gender & $\bar{n}$ & Mean & S.D. & $t$ & df & $\bar{p}$ \\
\hline Bodily/Kinesthetic & $\begin{array}{c}\text { Female } \\
\text { Male }\end{array}$ & $\begin{array}{c}96 \\
155\end{array}$ & $\begin{array}{l}8,01 \\
8,81\end{array}$ & $\begin{array}{l}5,07 \\
5,13\end{array}$ & $-1,219$ & 249 & ,224 \\
\hline Existential & $\begin{array}{c}\text { Female } \\
\text { Male }\end{array}$ & $\begin{array}{c}96 \\
155\end{array}$ & $\begin{array}{l}8,60 \\
9,69\end{array}$ & $\begin{array}{l}4,40 \\
5,32\end{array}$ & 1,760 & 229,047 & ,080 \\
\hline Interpersonal/Social & $\begin{array}{c}\text { Female } \\
\text { Male }\end{array}$ & $\begin{array}{c}96 \\
155\end{array}$ & $\begin{array}{c}8,97 \\
11,16\end{array}$ & $\begin{array}{l}4,97 \\
5,41\end{array}$ & $-3,198$ & 249 &, $002^{*}$ \\
\hline Intrapersonal & $\begin{array}{c}\text { Female } \\
\text { Male }\end{array}$ & $\begin{array}{c}96 \\
155\end{array}$ & $\begin{array}{l}7,56 \\
9,32 \\
\end{array}$ & $\begin{array}{l}3,98 \\
5,56\end{array}$ & $-2,913$ & 243,762 & ,004* \\
\hline Logical/Mathematical & $\begin{array}{c}\text { Female } \\
\text { Male }\end{array}$ & $\begin{array}{c}96 \\
155 \\
\end{array}$ & $\begin{array}{l}7,33 \\
9,96 \\
\end{array}$ & $\begin{array}{l}4,87 \\
5,46 \\
\end{array}$ & $-3,863$ & 249 & ,OOO* \\
\hline $\begin{array}{l}\text { Musical- } \\
\text { Rhythmic/Harmonic }\end{array}$ & $\begin{array}{l}\text { Female } \\
\text { Male }\end{array}$ & $\begin{array}{c}96 \\
155 \\
\end{array}$ & $\begin{array}{l}11,37 \\
14,63 \\
\end{array}$ & $\begin{array}{l}5,99 \\
5,90 \\
\end{array}$ & $-4,224$ & 249 & ,OOO* \\
\hline Naturalistic & $\begin{array}{c}\text { Female } \\
\text { Male }\end{array}$ & $\begin{array}{c}96 \\
155 \\
\end{array}$ & $\begin{array}{c}8,93 \\
12,14 \\
\end{array}$ & $\begin{array}{l}5,89 \\
6,06 \\
\end{array}$ & $-4,118$ & 249 & ,OOO* \\
\hline Verbal/Linguistic & $\begin{array}{c}\text { Female } \\
\text { Male }\end{array}$ & $\begin{array}{c}96 \\
155 \\
\end{array}$ & $\begin{array}{l}14,88 \\
18,31 \\
\end{array}$ & $\begin{array}{l}5,94 \\
6,10 \\
\end{array}$ & $-4,371$ & 249 & ,OOO* \\
\hline Visual/Spatial & $\begin{array}{c}\text { Female } \\
\text { Male }\end{array}$ & $\begin{array}{c}96 \\
155\end{array}$ & $\begin{array}{l}11,96 \\
14,91\end{array}$ & $\begin{array}{l}5,35 \\
5,76\end{array}$ & $-4,113$ & 212,702 & ,OOO* \\
\hline
\end{tabular}

When Table 4 is analyzed, significant differences were found between grades in terms of existential, intrapersonal, logical/mathematical, Musical-Rhythmic/Harmonic, naturalistic, verbal and visual intelligence ( $p$ $<0.05)$. It has been determined that bodily/kinesthetic and interpersonal/social intelligence do not change according to grades ( $>$ > 0.05). Third-year students reported to be more dominant in every intelligence area. In the areas of visual and existential intelligence, both third year and final year students were more dominant than students in other grades.

Sub-problem 4: Do the intelligence areas of the students differ significantly according to the departments?

When Table 5 is examined, there are no significant differences between departments in terms of multiple intelligence modalities $(\mathrm{p}>0,05)$.

Sub-problem 5:_Do students have age group differences in multiple intelligence areas?

When Table 6 is examined, visual/spatial intelligence shows age group differences in the favor of the students at 30 years and above. There were no significant age group differences in other intelligence areas.

Sub-problem 6: Do students have sport age group differences in multiple intelligence areas? 


\begin{tabular}{|c|c|c|c|c|c|c|c|}
\hline Modality & Grade & $\mathbf{n}$ & Mean & S.D. & f & $\mathrm{p}$ & Post Hoc \\
\hline Bodily/Kinesthetic & $\begin{array}{c}\text { Fresher } \\
\text { Sophomore } \\
\text { Third year } \\
\text { Final year }\end{array}$ & $\begin{array}{l}84 \\
26 \\
80 \\
61\end{array}$ & $\begin{array}{l}8,78 \\
8,80 \\
7,93 \\
8,75 \\
\end{array}$ & $\begin{array}{l}5,49 \\
4,15 \\
4,76 \\
5,43 \\
\end{array}$ & , 488 & ,691 & \\
\hline Existential & $\begin{array}{c}\text { Fresher } \\
\text { Sophomore } \\
\text { Third year } \\
\text { Final year }\end{array}$ & $\begin{array}{l}84 \\
26 \\
80 \\
61 \\
\end{array}$ & $\begin{array}{c}10,84 \\
8,61 \\
8,33 \\
8,63 \\
\end{array}$ & $\begin{array}{l}5,46 \\
5,54 \\
4,07 \\
4,85 \\
\end{array}$ & 4,320 & ,005* & $\begin{array}{l}3>1 \\
4>1\end{array}$ \\
\hline Interpersonal/Social & $\begin{array}{c}\text { Fresher } \\
\text { Sophomore } \\
\text { Third year } \\
\text { Final year }\end{array}$ & $\begin{array}{l}84 \\
26 \\
80 \\
61\end{array}$ & $\begin{array}{c}11,19 \\
11,26 \\
9,51 \\
9,80\end{array}$ & $\begin{array}{l}5,45 \\
5,60 \\
5,30 \\
5,03\end{array}$ & 1,830 &, 142 & \\
\hline Intrapersonal & $\begin{array}{c}\text { Fresher } \\
\text { Sophomore } \\
\text { Third year } \\
\text { Final year }\end{array}$ & $\begin{array}{l}84 \\
26 \\
80 \\
61 \\
\end{array}$ & $\begin{array}{c}10,17 \\
9,19 \\
7,02 \\
8,44 \\
\end{array}$ & $\begin{array}{l}5,44 \\
5,29 \\
3,52 \\
5,62 \\
\end{array}$ & 5,692 & ,001* & $3>1$ \\
\hline Logical/Mathematical & $\begin{array}{c}\text { Fresher } \\
\text { Sophomore } \\
\text { Third year } \\
\text { Final year }\end{array}$ & $\begin{array}{l}84 \\
26 \\
80 \\
61 \\
\end{array}$ & $\begin{array}{c}10,01 \\
8,61 \\
7,33 \\
9,78 \\
\end{array}$ & $\begin{array}{l}4,91 \\
5,72 \\
5,10 \\
5,83 \\
\end{array}$ & 4,140 &, $007 *$ & $\begin{array}{l}3>1 \\
3>4\end{array}$ \\
\hline $\begin{array}{l}\text { Musical- } \\
\text { Rhythmic/Harmonic }\end{array}$ & $\begin{array}{l}\text { Fresher } \\
\text { Sophomore } \\
\text { Third year } \\
\text { Final year }\end{array}$ & $\begin{array}{l}84 \\
26 \\
80 \\
61\end{array}$ & $\begin{array}{l}14,82 \\
14,53 \\
10,76 \\
14,36\end{array}$ & $\begin{array}{l}5,55 \\
5,47 \\
5,65 \\
6,77\end{array}$ & 7,823 & ,OOO* & $\begin{array}{l}3>1 \\
3>2 \\
3>4\end{array}$ \\
\hline Naturalistic & $\begin{array}{c}\text { Fresher } \\
\text { Sophomore } \\
\text { Third year } \\
\text { Final year }\end{array}$ & $\begin{array}{l}84 \\
26 \\
80 \\
61 \\
\end{array}$ & $\begin{array}{c}12,78 \\
11,80 \\
8,92 \\
10,59 \\
\end{array}$ & $\begin{array}{l}5,90 \\
6,49 \\
5,90 \\
6,11 \\
\end{array}$ & 5,871 &, $001^{*}$ & $3>1$ \\
\hline Verbal/Linguistic & $\begin{array}{c}\text { Fresher } \\
\text { Sophomore } \\
\text { Third year } \\
\text { Final year }\end{array}$ & $\begin{array}{l}84 \\
26 \\
80 \\
61 \\
\end{array}$ & $\begin{array}{l}18,15 \\
18,19 \\
14,63 \\
18,01 \\
\end{array}$ & $\begin{array}{l}6,48 \\
5,28 \\
5,87 \\
6,08 \\
\end{array}$ & 5,935 &, $001^{*}$ & $\begin{array}{l}3>1 \\
3>2 \\
3>4 \\
\end{array}$ \\
\hline Visual/Spatial & $\begin{array}{c}\text { Fresher } \\
\text { Sophomore } \\
\text { Third year } \\
\text { Final year }\end{array}$ & $\begin{array}{l}84 \\
26 \\
80 \\
61\end{array}$ & $\begin{array}{l}15,71 \\
13,69 \\
12,41 \\
12,98\end{array}$ & $\begin{array}{l}5,98 \\
4,53 \\
5,63 \\
5,57\end{array}$ & 5,266 &, $002^{*}$ & $\begin{array}{l}3>1 \\
4>1\end{array}$ \\
\hline
\end{tabular}

Note: $\mathrm{p}<0.05$

Table-5. Differences between departments in terms of multiple intelligence.

\begin{tabular}{|c|c|c|c|c|c|c|}
\hline Modality & Department & $\mathbf{N}$ & Mean & S.D. & $\mathrm{f}$ & p \\
\hline Bodily/Kinesthetic & $\begin{array}{l}\text { Coaching Education } \\
\text { Physical Education and Sports Teaching } \\
\text { Sport Management }\end{array}$ & $\begin{array}{c}113 \\
96 \\
42\end{array}$ & $\begin{array}{l}9,17 \\
8,29 \\
7,21\end{array}$ & $\begin{array}{l}5,62 \\
4,75 \\
4,19\end{array}$ & 2,422 & ,091 \\
\hline Existential & $\begin{array}{l}\text { Coaching Education } \\
\text { Physical Education and Sports Teaching } \\
\text { Sport Management }\end{array}$ & $\begin{array}{c}113 \\
96 \\
42 \\
\end{array}$ & $\begin{array}{l}9,62 \\
9,22 \\
8,45 \\
\end{array}$ & $\begin{array}{l}5,12 \\
5,00 \\
4,73 \\
\end{array}$ &, 849 & ,429 \\
\hline Interpersonal/Social & $\begin{array}{l}\text { Coaching Education } \\
\text { Physical Education and Sports Teaching } \\
\text { Sport Management }\end{array}$ & $\begin{array}{c}113 \\
96 \\
42 \\
\end{array}$ & $\begin{array}{c}10,72 \\
10,18 \\
9,57 \\
\end{array}$ & $\begin{array}{l}5,73 \\
5,33 \\
4,20 \\
\end{array}$ &, 764 & ,467 \\
\hline Intrapersonal & $\begin{array}{l}\text { Coaching Education } \\
\text { Physical Education and Sports Teaching } \\
\text { Sport Management }\end{array}$ & $\begin{array}{c}113 \\
96 \\
42\end{array}$ & $\begin{array}{l}8,91 \\
8,46 \\
8,35\end{array}$ & $\begin{array}{l}5,57 \\
4,39 \\
5,24\end{array}$ &, 279 &, 757 \\
\hline Logical/Mathematical & $\begin{array}{l}\text { Coaching Education } \\
\text { Physical Education and Sports Teaching } \\
\text { Sport Management }\end{array}$ & $\begin{array}{c}113 \\
96 \\
42 \\
\end{array}$ & $\begin{array}{l}9,53 \\
8,48 \\
8,47 \\
\end{array}$ & $\begin{array}{l}5,82 \\
5,13 \\
4,69 \\
\end{array}$ & 1,188 & ,306 \\
\hline $\begin{array}{l}\text { Musical- } \\
\text { Rhythmic/Harmonic }\end{array}$ & $\begin{array}{l}\text { Coaching Education } \\
\text { Physical Education and Sports Teaching } \\
\text { Sport Management }\end{array}$ & $\begin{array}{c}113 \\
96 \\
42 \\
\end{array}$ & $\begin{array}{l}13,90 \\
13,07 \\
12,71 \\
\end{array}$ & $\begin{array}{l}5,74 \\
6,45 \\
6,40 \\
\end{array}$ &, 776 & ,461 \\
\hline Naturalistic & $\begin{array}{l}\text { Coaching Education } \\
\text { Physical Education and Sports Teaching } \\
\text { Sport Management }\end{array}$ & $\begin{array}{c}113 \\
96 \\
42\end{array}$ & $\begin{array}{l}10,90 \\
10,11 \\
12,80\end{array}$ & $\begin{array}{l}5,98 \\
5,77 \\
7,30\end{array}$ & 2,808 & ,062 \\
\hline Verbal/Linguistic & $\begin{array}{l}\text { Coaching Education } \\
\text { Physical Education and Sports Teaching } \\
\text { Sport Management }\end{array}$ & $\begin{array}{c}113 \\
96 \\
42 \\
\end{array}$ & $\begin{array}{l}17,15 \\
16,65 \\
17,38 \\
\end{array}$ & $\begin{array}{l}6,04 \\
6,47 \\
6,43 \\
\end{array}$ &, 258 &, 773 \\
\hline Visual/Spatial & $\begin{array}{l}\text { Coaching Education } \\
\text { Physical Education and Sports Teaching } \\
\text { Sport Management }\end{array}$ & $\begin{array}{c}113 \\
96 \\
42 \\
\end{array}$ & $\begin{array}{l}13,66 \\
13,56 \\
14,64 \\
\end{array}$ & $\begin{array}{l}5,44 \\
5,90 \\
6,44 \\
\end{array}$ &, 556 &, 574 \\
\hline
\end{tabular}

Note: $p<0.05$ 
Table-6. Differences between age groups in terms of multiple intelligence.

\begin{tabular}{|c|c|c|c|c|c|c|}
\hline Modality & Age Group & $\mathbf{n}$ & Mean & S.D. & $f$ & $\mathbf{p}$ \\
\hline Bodily/Kinesthetic & $\begin{array}{l}18 \text { years and under } \\
\text { Between } 19 \text { and } 22 \text { years } \\
\text { Between } 23 \text { and } 26 \text { years } \\
\text { Between } 27 \text { and } 29 \text { years } \\
30 \text { years and above }\end{array}$ & $\begin{array}{c}32 \\
177 \\
34 \\
1 \\
7 \\
\end{array}$ & $\begin{array}{c}7,68 \\
8,23 \\
10,29 \\
13,00 \\
10,00 \\
\end{array}$ & $\begin{array}{c}4,83 \\
4,90 \\
5,76 \\
- \\
7,14 \\
\end{array}$ & 1,733 &, 143 \\
\hline Existential & $\begin{array}{l}18 \text { years and under } \\
\text { Between } 19 \text { and } 22 \text { years } \\
\text { Between } 23 \text { and } 26 \text { years } \\
\text { Between } 27 \text { and } 29 \text { years } \\
\text { 30 years and above }\end{array}$ & $\begin{array}{c}32 \\
177 \\
34 \\
1 \\
7 \\
\end{array}$ & $\begin{array}{l}11,53 \\
8,92 \\
9,23 \\
7,00 \\
8,42 \\
\end{array}$ & $\begin{array}{c}5,24 \\
4,91 \\
4,75 \\
- \\
6,37 \\
\end{array}$ & 1,965 &, 100 \\
\hline Interpersonal/Social & $\begin{array}{l}18 \text { years and under } \\
\text { Between } 19 \text { and } 22 \text { years } \\
\text { Between } 23 \text { and } 26 \text { years } \\
\text { Between } 27 \text { and } 29 \text { years } \\
30 \text { years and above }\end{array}$ & $\begin{array}{c}32 \\
177 \\
34 \\
1 \\
7 \\
\end{array}$ & $\begin{array}{l}11,25 \\
10,20 \\
10,02 \\
14,00 \\
10,14 \\
\end{array}$ & $\begin{array}{c}5,59 \\
5,15 \\
5,97 \\
- \\
6,86 \\
\end{array}$ & ,404 &, 806 \\
\hline Intrapersonal & $\begin{array}{l}18 \text { years and under } \\
\text { Between } 19 \text { and } 22 \text { years } \\
\text { Between } 23 \text { and } 26 \text { years } \\
\text { Between } 27 \text { and } 29 \text { years } \\
30 \text { years and above }\end{array}$ & $\begin{array}{c}32 \\
177 \\
34 \\
1 \\
7 \\
\end{array}$ & $\begin{array}{l}11,00 \\
8,25 \\
8,41 \\
7,00 \\
9,28 \\
\end{array}$ & $\begin{array}{c}5,47 \\
4,73 \\
5,12 \\
- \\
9,14 \\
\end{array}$ & 2,086 & ,083 \\
\hline Logical/Mathematical & $\begin{array}{l}18 \text { years and under } \\
\text { Between } 19 \text { and } 22 \text { years } \\
\text { Between } 23 \text { and } 26 \text { years } \\
\text { Between } 27 \text { and } 29 \text { years } \\
30 \text { years and above } \\
\end{array}$ & $\begin{array}{c}32 \\
177 \\
34 \\
1 \\
7 \\
\end{array}$ & $\begin{array}{c}10,65 \\
8,48 \\
9,82 \\
7,00 \\
9,42 \\
\end{array}$ & $\begin{array}{c}5,12 \\
5,20 \\
5,81 \\
- \\
8,24 \\
\end{array}$ & 1,414 &, 230 \\
\hline $\begin{array}{l}\text { Musical- } \\
\text { Rhythmic/Harmonic }\end{array}$ & $\begin{array}{l}18 \text { years and under } \\
\text { Between } 19 \text { and } 22 \text { years } \\
\text { Between } 23 \text { and } 26 \text { years } \\
\text { Between } 27 \text { and } 29 \text { years } \\
30 \text { years and above }\end{array}$ & $\begin{array}{c}32 \\
177 \\
34 \\
1 \\
7 \\
\end{array}$ & $\begin{array}{l}15,65 \\
12,76 \\
14,52 \\
16,00 \\
12,85 \\
\end{array}$ & $\begin{array}{c}5,28 \\
6,21 \\
6,05 \\
- \\
6,44 \\
\end{array}$ & 1,935 &, 105 \\
\hline Naturalistic & $\begin{array}{l}18 \text { years and under } \\
\text { Between } 19 \text { and } 22 \text { years } \\
\text { Between } 23 \text { and } 26 \text { years } \\
\text { Between } 27 \text { and } 29 \text { years } \\
\text { 30 years and above }\end{array}$ & $\begin{array}{c}32 \\
177 \\
34 \\
1 \\
7 \\
\end{array}$ & $\begin{array}{c}12,62 \\
10,55 \\
11,58 \\
7,00 \\
9,71 \\
\end{array}$ & $\begin{array}{c}6,13 \\
6,14 \\
6,54 \\
- \\
5,93 \\
\end{array}$ & 1,028 & ,394 \\
\hline Verbal/Linguistic & $\begin{array}{l}18 \text { years and under } \\
\text { Between } 19 \text { and } 22 \text { years } \\
\text { Between } 23 \text { and } 26 \text { years } \\
\text { Between } 27 \text { and } 29 \text { years } \\
30 \text { years and above }\end{array}$ & $\begin{array}{c}32 \\
177 \\
34 \\
1 \\
7 \\
\end{array}$ & $\begin{array}{l}18,37 \\
16,53 \\
18,55 \\
15,00 \\
15,42 \\
\end{array}$ & $\begin{array}{c}6,45 \\
6,24 \\
5,43 \\
- \\
8,71 \\
\end{array}$ & 1,304 & ,269 \\
\hline Visual/Spatial & $\begin{array}{l}18 \text { years and under } \\
\text { Between } 19 \text { and } 22 \text { years } \\
\text { Between } 23 \text { and } 26 \text { years } \\
\text { Between } 27 \text { and } 29 \text { years } \\
30 \text { years and above }\end{array}$ & $\begin{array}{c}32 \\
177 \\
34 \\
1 \\
7\end{array}$ & $\begin{array}{l}17,06 \\
13,29 \\
13,97 \\
11,00 \\
10,71\end{array}$ & $\begin{array}{c}5,97 \\
5,64 \\
4,92 \\
- \\
8,05\end{array}$ & 3,583 &, 007 \\
\hline
\end{tabular}

Note: $\mathrm{p}<0.05$, Groups with only one case are ignored in computing the test of homogeneity of variance for.

Table-7. Differences between sport age groups in terms of multiple intelligence.

\begin{tabular}{|c|c|c|c|c|c|c|c|}
\hline Modality & Sport Year & $\mathbf{n}$ & Mean & S.D. & $\mathrm{f}$ & $\mathbf{p}$ & Post Hoc \\
\hline Bodily/Kinesthetic & $\begin{array}{l}5 \text { years and under } \\
\text { Between } 6-10 \text { years } \\
\text { Between } 11-15 \text { years } \\
16 \text { years and above }\end{array}$ & \begin{tabular}{c|}
52 \\
151 \\
28 \\
20 \\
\end{tabular} & $\begin{array}{c}8,30 \\
8,52 \\
10,03 \\
6,80 \\
\end{array}$ & $\begin{array}{l}5,23 \\
5,21 \\
5,22 \\
3,22 \\
\end{array}$ & 1,614 &, 187 & \\
\hline Existential & $\begin{array}{l}5 \text { years and under } \\
\text { Between } 6-10 \text { years } \\
\text { Between } 11-15 \text { years } \\
16 \text { years and above }\end{array}$ & \begin{tabular}{c|}
52 \\
151 \\
28 \\
20 \\
\end{tabular} & $\begin{array}{c}10,26 \\
9,33 \\
9,39 \\
6,15 \\
\end{array}$ & $\begin{array}{l}5,31 \\
5,03 \\
4,71 \\
3,21 \\
\end{array}$ & 3,375 & ,019* & $\begin{array}{c}16>5 \\
16>6-10\end{array}$ \\
\hline Interpersonal/Social & $\begin{array}{l}5 \text { years and under } \\
\text { Between } 6-10 \text { years } \\
\text { Between } 11-15 \text { years } \\
16 \text { years and above }\end{array}$ & \begin{tabular}{c|}
52 \\
151 \\
28 \\
20 \\
\end{tabular} & $\begin{array}{c}10,84 \\
10,64 \\
11,03 \\
5,60 \\
\end{array}$ & $\begin{array}{l}4,93 \\
5,35 \\
5,52 \\
3,92 \\
\end{array}$ & 6,054 & ,001* & $\begin{array}{c}16>5 \\
16>6-10 \\
16>11-15\end{array}$ \\
\hline Intrapersonal & $\begin{array}{l}5 \text { years and under } \\
\text { Between } 6-10 \text { years } \\
\text { Between } 11-15 \text { years } \\
16 \text { years and above }\end{array}$ & \begin{tabular}{c|}
52 \\
151 \\
28 \\
20 \\
\end{tabular} & $\begin{array}{l}9,00 \\
8,82 \\
9,71 \\
4,95 \\
\end{array}$ & $\begin{array}{l}6,06 \\
4,85 \\
4,57 \\
2,96 \\
\end{array}$ & 4,238 & ,006* & $\begin{array}{c}16>5 \\
16>6-10 \\
16>11-15\end{array}$ \\
\hline Logical/Mathematical & $\begin{array}{l}5 \text { years and under } \\
\text { Between } 6-10 \text { years } \\
\text { Between } 11-15 \text { years } \\
16 \text { years and above }\end{array}$ & \begin{tabular}{c|}
52 \\
151 \\
28 \\
20 \\
\end{tabular} & $\begin{array}{l}9,50 \\
9,16 \\
9,75 \\
4,90 \\
\end{array}$ & $\begin{array}{l}6,25 \\
5,08 \\
5,16 \\
4,02 \\
\end{array}$ & 4,395 & ,005* & $\begin{array}{c}16>5 \\
16>6-10 \\
16>11-15\end{array}$ \\
\hline Musical-Rhythmic/Harmonic & $\begin{array}{l}5 \text { years and under } \\
\text { Between } 6-10 \text { years } \\
\text { Between } 11-15 \text { years } \\
16 \text { years and above } \\
\end{array}$ & \begin{tabular}{c|}
52 \\
151 \\
28 \\
20 \\
\end{tabular} & $\begin{array}{c}12,94 \\
13,74 \\
14,89 \\
9,75 \\
\end{array}$ & $\begin{array}{l}6,38 \\
5,93 \\
6,10 \\
5,94 \\
\end{array}$ & 3,252 &, $022^{*}$ & $\begin{array}{c}16>6-10 \\
16>11-15\end{array}$ \\
\hline Naturalistic & $\begin{array}{l}5 \text { years and under } \\
\text { Between } 6-10 \text { years } \\
\text { Between } 11-15 \text { years } \\
16 \text { years and above } \\
\end{array}$ & $\begin{array}{c}52 \\
151 \\
28 \\
20 \\
\end{array}$ & $\begin{array}{c}11,53 \\
10,87 \\
12,64 \\
7,25 \\
\end{array}$ & $\begin{array}{l}6,40 \\
6,40 \\
5,16 \\
3,62 \\
\end{array}$ & 3,331 & ,020* & $\begin{array}{c}16>5 \\
16>11-15\end{array}$ \\
\hline Verbal/Linguistic & $\begin{array}{l}5 \text { years and under } \\
\text { Between } 6-10 \text { years } \\
\text { Between } 11-15 \text { years } \\
16 \text { years and above } \\
\end{array}$ & $\begin{array}{c}52 \\
151 \\
28 \\
20 \\
\end{array}$ & $\begin{array}{l}15,51 \\
17,07 \\
20,64 \\
15,25 \\
\end{array}$ & $\begin{array}{l}7,57 \\
5,96 \\
4,58 \\
4,73 \\
\end{array}$ & 4,877 & , $003^{*}$ & $\begin{array}{c}11-15>5 \\
11-15>6-10 \\
16>11-15 \\
\end{array}$ \\
\hline Visual/Spatial & $\begin{array}{l}5 \text { years and under } \\
\text { Between } 6-10 \text { years } \\
\text { Between } 11-15 \text { years } \\
16 \text { years and above }\end{array}$ & \begin{tabular}{c|}
52 \\
151 \\
28 \\
20 \\
\end{tabular} & $\begin{array}{l}12,90 \\
14,06 \\
15,46 \\
11,65\end{array}$ & $\begin{array}{l}6,39 \\
5,67 \\
5,19 \\
5,13 \\
\end{array}$ & 2,250 & ,083 & \\
\hline
\end{tabular}


In Table 7, significant differences were found between sport age groups in terms of existential, interpersonal/social, intrapersonal, logical/mathematical, Musical-Rhythmic/Harmonic, naturalistic, and verbal intelligence $(p<0,05)$. No significant differences were found in terms of bodily/kinesthetic intelligence and visual/spatial intelligence ( $p>0,05)$. The students doing sport for 16 years and more reported to have higher scores in each modality. In verbal intelligence area, the students doing sport between 11-15 years and for 16 years and above had higher scores than those doing sport lesser time.

Sub-problem 7: Do students have sport branch differences in multiple intelligence areas?

Table-8. Differences between students according to their branches in terms of multiple intelligence.

\begin{tabular}{|c|c|c|c|c|c|c|c|}
\hline Modality & Branch & $\bar{n}$ & Mean & S.D. & $\mathrm{t}$ & df & $\bar{p}$ \\
\hline Bodily/Kinesthetic & $\begin{array}{l}\text { Team } \\
\text { Individual }\end{array}$ & $\begin{array}{l}135 \\
116\end{array}$ & $\begin{array}{l}8,78 \\
8,18\end{array}$ & $\begin{array}{l}5,09 \\
5,14\end{array}$ &, 919 & 249 & ,359 \\
\hline Existential & $\begin{array}{l}\text { Team } \\
\text { Individual }\end{array}$ & $\begin{array}{l}135 \\
116\end{array}$ & $\begin{array}{l}9,65 \\
8,84\end{array}$ & $\begin{array}{l}5,08 \\
4,91\end{array}$ & 1,273 & 249 & ,204 \\
\hline Interpersonal/Social & $\begin{array}{l}\text { Team } \\
\text { Individual }\end{array}$ & $\begin{array}{l}135 \\
116\end{array}$ & $\begin{array}{l}10,25 \\
10,41\end{array}$ & $\begin{array}{l}4,91 \\
5,83\end{array}$ &,- 236 & 225,793 & ,814 \\
\hline Intrapersonal & $\begin{array}{l}\text { Team } \\
\text { Individual }\end{array}$ & $\begin{array}{l}135 \\
116 \\
\end{array}$ & $\begin{array}{l}7,56 \\
9,32 \\
\end{array}$ & $\begin{array}{l}5,24 \\
4,91 \\
\end{array}$ & ,058 & 249 & ,954 \\
\hline Logical/Mathematical & $\begin{array}{l}\text { Team } \\
\text { Individual }\end{array}$ & $\begin{array}{l}135 \\
116\end{array}$ & $\begin{array}{l}7,33 \\
9,96\end{array}$ & $\begin{array}{l}5,32 \\
5,48\end{array}$ &,- 554 & 249 &, 580 \\
\hline Musical-Rhythmic/Harmonic & $\begin{array}{l}\text { Team } \\
\text { Individual }\end{array}$ & $\begin{array}{l}135 \\
116 \\
\end{array}$ & $\begin{array}{l}11,37 \\
14,63 \\
\end{array}$ & $\begin{array}{l}6,26 \\
5,99\end{array}$ &, 532 & 249 &, 595 \\
\hline Naturalistic & $\begin{array}{l}\text { Team } \\
\text { Individual }\end{array}$ & $\begin{array}{l}135 \\
116 \\
\end{array}$ & $\begin{array}{l}11,25 \\
10,53 \\
\end{array}$ & $\begin{array}{l}6,39 \\
5,95 \\
\end{array}$ &, 915 & 249 &, 361 \\
\hline Verbal/Linguistic & $\begin{array}{l}\text { Team } \\
\text { Individual }\end{array}$ & $\begin{array}{l}135 \\
116 \\
\end{array}$ & $\begin{array}{l}17,31 \\
16,63 \\
\end{array}$ & $\begin{array}{l}6,53 \\
5,92 \\
\end{array}$ &, 858 & 249 & ,391 \\
\hline Visual/Spatial & $\begin{array}{l}\text { Team } \\
\text { Individual }\end{array}$ & $\begin{array}{l}135 \\
116\end{array}$ & $\begin{array}{l}13,97 \\
13,57\end{array}$ & $\begin{array}{l}6,16 \\
5,32\end{array}$ & ,536 & 249 & ,593 \\
\hline
\end{tabular}

Note: $\mathrm{p}<0.05$

Table 8 presents the differences between sport branches in terms of multiple intelligence. No significant differences were found between sport branches.

\section{Discussion and Conclusion}

The aim of this study was to determine the dominant intelligence modality of university students who receive sports education and to examine intelligence modality in terms of various variables (gender, grade, department, age, sport age, and branch). In this section, the results obtained from the research findings and the relationship of these results with the relevant literature were discussed and suggestions were made for other studies to be conducted.

The first hypothesis was "While the most dominant intelligence field of students studying in the field of sports sciences is physical / kinesthetic intelligence, the most distant intelligence field is verbal intelligence." The intelligence area which students are the most dominant is the physical intelligence area with an average of 8.51. The intelligence area which the students are most distant is the verbal intelligence area with an average of 17.00. This hypothesis was accepted. In the studies of Bayrak et al. (2005); Hoşgörür and Katrancı (2007) and Kul et al. (2014) the field of intelligence in which physical education and sports school students are dominant was physical / kinesthetic intelligence and verbal intelligence was the area of intelligence they are most distant from. Some studies supported our results (Baba. \& Güçlü, 2015; Kiremitçi \& Canpolat, 2014; Kul et al., 2014; Oral, 2001).

In the study of Ermiş et al. (2018) it was stated that the lowest intelligence field is verbal intelligence in adolescents who do sports and do not do sports. According to a different study on the intelligence type of Turkish language teaching students (Eyyam, Meneviş, \& Doğruer, 2010) there was a significant difference between students' verbal multiple intelligence and their academic achievements. In addition, individuals who successfully achieve their physical goals and control their movements are in this intelligence sphere. Skills such as strength, balance, speed, flexibility, coordination and dexterity are seen in this intelligence field. Actors, artisans, dancers, athletes and sculptors can be given as master examples of physical intelligence. The person's ability to use his body while expressing his ideas or feelings expresses this field of intelligence (Armstrong, 2003, 2009; Babacan \& Dilci, 2012; Gardner, 1999; Moran, Kornhaber, \& Gardner, 2006; Nolen, 2003). Likewise, because of the more intensive lessons to develop bodily / kinesthetic intelligence in institutions providing education about physical education, students studying in these schools are expected to have higher bodily / kinesthetic intelligence (Baba. \& Güçlü, 2015).

The second hypothesis was "There are significant differences in multiple intelligence areas according to the students' gender." There were significant differences between genders regarding interpersonal/social, intrapersonal, logical/mathematical, Musical-Rhythmic/Harmonic, naturalistic, verbal and visual intelligence areas $(\mathrm{p}<0.05)$. This hypothesis was accepted. Females were more dominant than males in terms of each modality. Some studies in literature supported our findings reporting significant differences (Azar, 2006; Baba et al., 2015; Cinkılıç \& Soyer, 2013; Furnham \& Chamorro-Premuzic, 2005) and insignificance between genders in terms of some multiple intelligence modalities (Kahraman \& Bavlı, 2014; Kahraman. \& Bulut, 2014; Loori, 2005; Metan \& Küçük, 2017; Neville, 2000; Yenice \& Aktamış, 2010). In addition, in the study of Altınok (2008) and Ermiş et al. (2018) the bodily / kinesthetic intelligence of women was higher than that of men, in line with the findings of our research. Senel and Yildız (2016) reported no significant gender differences in terms of bodily/kinesthetic intelligence. Similar to our findings, in the study conducted by Tunç (2008) there were significant differences between the genders in terms of verbal, visual, musical, interpersonal and intrapersonal intelligence modalities. In Yenilmez and Çalışkan (2011) study, there were significant differences in the areas of verbal, visual and musical intelligence 
according to the gender variable. In the studies of Tekin (2007); Tekin (2008) and Ylldiz (2010) the average of bodily / kinesthetic intelligence of men was higher than that of women. In addition, in the study of Tekin (2008) bodily / kinesthetic intelligence differs significantly according to the gender variable. Unlike these studies, no significant difference was found in the areas of gender and multiple intelligence in the study of Izci, Kara, and Dalaman (2007). The higher interpersonal intelligence of women is due to two reasons, according to Ciarrochi, Chan, and Caputi (2000). First, women are more social and can read other people's feelings better. Second, they are biologically better equipped for such a situation. In addition to this situation, Mayer, Caruso, and Salovey (1999) stated that women should read emotions better because they have less power in society. According to the opinions of Mayer et al. (1999) the fact that women are more dominant in terms of intrapersonal and logical intelligence can be based on this reason. In addition, women become socialized by taking an emotional attitude towards some of their difficulties (Zakowski et al., 2003). Women can share the difficult situations they face in life more easily than men. In addition, this may be associated with gender roles (Kimura, 2002). Therefore, it can be considered normal for women to have multiple intelligence levels more dominant than men.

The third hypothesis was 'There are significant differences in multiple intelligence areas according to students' grade levels." Significant differences were found between grades in terms of existential, intrapersonal, logical/mathematical, Musical-Rhythmic/Harmonic, naturalistic, verbal and visual intelligence $(\mathrm{p}<0.05)$. It has been determined that bodily/kinesthetic and interpersonal/social intelligence do not change according to grades ( $p>0.05)$. Third-year students reported to be more dominant in every intelligence area. In the areas of visual and existential intelligence, both third year and final year students were more dominant than students in other grades. This hypothesis was accepted. Some studies supported our findings reporting no significant grade differences in terms of bodily/kinesthetic intelligence (Altınok, 2008; Baba et al., 2015; Nulhakım, Wıbawa, \& Erwın, 2019; Tekin, 2007; Yenice \& Aktamış, 2010). In the study of Baba et al. (2015) the average of bodily / kinesthetic intelligence score of 3rd grade students was found higher than other grades. In the study of Güllü and Tekin (2009) significant differences were found in the areas of verbal, logical, interpersonal and intrapersonal intelligence according to grade. The reason for these differences can be shown as the differentiation of interests, wishes and expectations of students, which can change according to their grade levels (Güllü \& Tekin, 2009).

The fourth hypothesis was "There are significant differences in multiple intelligence areas according to the students' departments." there were no significant differences between departments in terms of multiple intelligence modalities $(p>0,05)$. This hypothesis was rejected. According to the study of Güllü and Tekin (2009) the intelligence areas of sports high school students and general high school students differed from each other. In the studies of Oztürkmen (2006) and Camurcu (2007) and Erman (2003) the intelligence areas in which students studying in different departments had different dominant intelligence modalities. Ermiş et al. (2012) compared the students of the Police Vocational School and the School of Physical Education and Sports in terms of intelligence and did not find a significant difference. The reason for insignificance between the departments in intelligence areas is that they take sports lessons at the same time, there is a special talent exam at the entrance to both departments and they like sports lessons (Ermiş et al., 2012). Also, Armstrong mentioned two factors that had a significant impact on intelligence development. These are accelerator and blinding factors. Accelerator factors have positive effects for intelligence development and blinding factors have negative effects (Kuru, 2001). In this context, it is a natural result that different intelligence fields develop as a result of different courses taken by students in different schools and departments.

The fifth hypothesis was "There are significant differences in multiple intelligence areas according to the students' ages." visual/spatial intelligence shows age group differences in the favor of the students at 30 years and above. There were no significant age group differences in other intelligence areas. This hypothesis was partially accepted. There are some studies in the literature that are similar to the findings of our research (Inan, Aydin, \& Bilgin, 2000; Metan \& Küçük, 2017). According to these studies, a decrease in reasoning skills was found with the increase in the age level. The reason for this decline has been shown that students are in a compulsory period for studying (Metan \& Küçük, 2017). In our study, it was seen that students in the exam preparation periods received lower averages. Elderly participants were found to have higher averages from their intelligence fields. There are different opinions supporting these findings. According to these views, as the age of the individual progresses, they gain different experiences and therefore their ability to use information increases with age (Alpar, 2000; Uysal, 2006). It is known that intelligence is influenced not only by heredity but also by the environmental factor with which the family and the individual interact. According to the multiple intelligence theory, the development of intelligence areas depends on providing opportunities and environment suitable for the individual rather than the existing natural capacity (Uysal, 2006).

The sixth hypothesis was "There are significant differences in the areas of multiple intelligence according to the duration of the students' sports." Significant differences were found between sport age groups in terms of existential, interpersonal/social, intrapersonal, logical/mathematical, Musical-Rhythmic/Harmonic, naturalistic, and verbal intelligence $(p<0,05)$. No significant differences were found in terms of bodily/kinesthetic intelligence and visual/spatial intelligence $(\mathrm{p}>0,05)$. The students doing sport for 16 years and more reported to have higher scores in each modality. In verbal intelligence area, the students doing sport between 11-15 years and for 16 years and above had higher scores than those doing sport lesser time. This hypothesis partially accepted. When the literature is examined, there are different research findings that are similar to the findings of our study (Cengiz \& Pulur, 2008; Demirsöz \& Kocabaş, 2006; Ermiş et al., 2012; Guastello \& Guastello, 2003; İlhan et al., 2005; Katz, Mirenda, \& Auerbach, 2002; Teele, 1997; Tekin, 2008; Tekin, Filiz, Tasgin, \& Özmutlu, 2008). In these studies, it was found that students who do sports are more dominant than students who do not do sports in some intelligence fields. In a study on the socialization levels of university students studying in different departments (Erdemir, Sert, \& Okmen, 2006) the levels of socialization of students of Physical Education and Sports were higher than those of the Music and Painting departments. According to the study of Canll, Toksöz, and Ozmutlu (2017) there is a significant and positive relationship between basketball-specific skill level and musical intelligence. It has been reported that sports have many positive effects on human development. Movement behavior and hence sport is an essential condition in individuals' development processes. Physical activity and sports in which the individual 
participates is very important in the cognitive, spiritual and emotional development of the individual (Gohla, 2010; Orhan, 2019). In addition, cognitive development starts at a very early age and is supported by physical activities, among other things. The development of thought structure and perception is closely related to motor abilities that require motion and sensory experience (Orhan, 2019; Zimmer, 2014).

The seventh hypothesis was "There are significant differences in multiple intelligence areas according to the branches that students make." No significant differences were found between sport branches in terms of multiple intelligence modalities. This hypothesis was rejected. There are different studies in the literature that support our research (Baba et al., 2015). According to these studies, no significant difference was found between the branch type and multiple intelligence areas in parallel with our research. Senel and Yildiz (2016) reported no significant differences between team and individual athletes in terms of bodily/kinesthetic intelligence. In the works of Altınok (2008) and Tekin (2007) bodily/kinesthetic intelligence of athletes engaged in individual sports was found higher than athletes doing team sports. In the study of Aslan et al. (2015) similar to our finding, the intrapersonal intelligence areas of athletes interested in team sports were more dominant. In Altınok (2008) study, a significant difference was found between the branch types in bodily/kinesthetic intelligence field.

Physical activity and sports that the individual participates contributes to cognitive, spiritual and emotional development (Gohla, 2010; Orhan, 2019). Since physical activity and sports make positive contributions to individuals 'multiple intelligence domains, participants' intelligence domains are close to each other. The fact that the branch type does not differ significantly in terms of multiple intelligence may be due to this reason.

Based on the findings of the research, the following suggestions can be made for future studies:

1) It will be appropriate to use a scale including 9 intelligence fields in future studies.

2) By doing longitudinal studies, developments in intelligence areas of the same individuals through sports can be brought to the literature in the light of current information.

3) Based on the results of the studies, applications can be developed to improve the intelligence areas where students are distant through sports and education, and the intelligence areas they are distant from.

4) Since it is known that multiple intelligence areas of individuals are affected by both personal and environmental factors, attention should be paid to creating the most appropriate educational environments.

5) The positive effects of sports on the development of multiple intelligence have been repeated with this study and therefore it is important that individuals are directed to sports for their intelligence development.

6) It has been determined in the literature that the verbal intelligence areas of the students receiving physical education and sports are low. Accordingly, it would be beneficial to make additional applications to improve the verbal intelligence field.

7) Wider sampling groups and different independent variables can also be proposed as an additional suggestion for future studies.

\section{References}

Alpar, A. M. (2000). Artificial intelligence and education. Retrieved from www.Tzv.Org. Tr. [Accessed 14.10.2004].

Altınok, E. (2008). Investigation of multiple intelligence areas of physical education students according to some variables. Unpublished Master Thesis, Selcuk University, Konya.

Armstrong, T. (2003). The multiple intelligences of reading and writing. Alexandria, VA: Association for Supervision and Curriculum Development.

Armstrong, T. (2009). Multiple intelligences in the classroom (3rd ed.). Alexandria: Association for Supervision and Curriculum Development.

Aslan, C. S., Dalkıran, O., \& Ozer, U. (2015). Investigation of multiple intelligence areas of athletes performing cyclic and non-cyclic sports, pilot study. International Journal of Contemporary Educational Research, 12(1), 26-33.

Azar, A. (2006). The relationship between the area selected in high school and OSS area scores and multiple intelligence profiles. Educational Administration: Theory and Practice, 46, 157-174.

Baba, K. H., Karakaş, G., \& Gizdem, S. (2015). Investigation of physical achievement and physical achievement of physical education and sports school students. Cbu Physical Journal of Physical Education and Sports, $10(1), 66-74$.

Baba., H., \& Güçlü, M. (2015). The effect of the kinesthetic intelligence, the emotional intelligence and interior-exterior control college of the students in physical education and sport academy to their academic achievement. The Online Journal of Recreation and Sport, 4(1).

Babacan, T., \& Dilci, T. (2012). Adaptation studies of multiple intelligence scale to Turkish. E-Journal Of Nerw World Sciences Academy NwesaEducation Sciences, 7(3), 969-982.

Başaran, B. (2004). Effective learning and multiple intelligence theory: A review. Aegean Education Magazine, 5(1).

Bayrak, C., Celiksoy, M. A., \& Celiksoy, S. (2005). The relationship between students' intelligence profiles and practice talent entrance examinations regarding multiple intelligence theory. Paper presented at the 4th National Physical Education and Sports Teaching Symposium. June 10-1 1, Bursa.

Camurcu, S. N. (2007). Determination of the distribution levels of primary level second level students regarding multiple intelligence areas. Unpublished Master Thesis. Trabzon: Karadeniz Technical University, Institute of Social Sciences.

Canlı, U., Toksöz, İ., \& Ozmutlu, İ. (2017). Evaluation of multiple intelligence areas, socio-economic levels and skills of basketball players. Journal of Human Sciences, 14(4), 4171-4181.

Cengiz, Ş., \& Pulur, A. (2008). The effect of 12-week football training on physical-kinesthetic and musical intelligence development of 8 -10 age group children. Niğde University Journal of Physical Education and Sports Sciences, 2(3).

Chongde, L., \& Tsingan, L. (2003). Multiple intelligence and the structure of thinking. Theory \& Psychology, 13(6), 829-845.Available at: https://doi.org/10.1177/0959354303136004.

Ciarrochi, J. V., Chan, A. Y., \& Caputi, P. (2000). A critical evaluation of the emotional intelligence construct. Personality and Individual Differences, 28(3), 539-561.Available at: https://doi.org/10.1016/s0191-8869(99)00119-1.

Cinkılıç, I., \& Soyer, F. (2013). Investigation of the relationship between multiple intelligence areas and problem solving skills of physical education teacher candidates. Journal of Sports Management and Information Technologies, 8(1).

Demirsöz, S. E., \& Kocabaş, A. (2006). The effects of cooperative learning based on multiple intelligence theories on the access and reminder of music. Paper presented at the Gazi University V. National Classroom Teaching Congress, Ankara.

Erdemir, İ., Sert, N., \& Okmen, A. S. (2006). Comparison of socialization levels of physical education and sports school, painting and music department students. Paper presented at the 9th International Congress of Sports Sciences Proceedings Book, Muğla.

Erman, A. (2003). Comparative analysis of the distribution and level measurements of multiple intelligence types of 4th grade primary school students with musical intelligence levels. Published Master Thesis. Ankara: Gazi University, Institute of Educational Sciences.

Ermiş, E., Ermiş, A., \& İmamoğlu, O. (2018). The relationship between sports and multiple intelligence in students during adulthood. Turkish Studies, 13/18, 589-598.

Ermiş, E., İmamoğlu, O., \& Erilli, N. A. (2012). The effect of sport on physical and social multiple intelligence points of university students. Journal of Sports and Performance Researches, 3(2). 
Eyyam, R., Meneviş, İ., \& Doğruer, N. (2010). The relationship between multiple intelligence and academic achievement. Paper presented at the International Conference on New Trends in Education and their Implications, 11-13 November, Antalya, Turkey.

Furnham, A., \& Chamorro-Premuzic, T. (2005). Estimating one's own and one's relatives' multiple intelligence: A study from Argentina. The Spanish Journal of Psychology, 8(1), 12-20.Available at: https://doi.org/10.1017/s1138741600004911.

Gardner, H. (1999). Intelligence reframed: Multiple intelligences for the 21 th century. New York: Basic Books.

Gohla, S. (2010). The importance of sport and exercise in child development. Administrative Portal.

Guastello, D. D., \& Guastello, S. J. (2003). Androgyny, gender role behavior, and emotional intelligence among college students and their parents. Sex Roles, 49(11-12), 663-673.Available at: https://doi.org/10.1023/b:sers.0000003136.67714.04.

Güllü, M., \& Tekin, M. (2009). Comparison of multiple intelligence areas of sports high school students and general high school students. Niğde University Journal of Physical Education and Sports Sciences, 3(3), 247-258.

Hoşgörür, V., \& Katrancı, M. (2007). Dominant intelligence areas of class and physical education and sports teaching students (Example of Kırıkkale University Education Faculty). Ondokuz Mayzs University Journal of Education Faculty, 24, 33-42.

İlhan, A., Mirzeoğlu, D., Aktaş, İ., \& Demir, V. (2005). The effect of gymnastics and volleyball units processed in line with multiple intelligence applications on students' cognitive and psycho-motor development. Journal of Spormeter Physical Education and Sports Sciences, 3(1), 5--10.

Inan, M., Aydin, O., \& Bilgin, H. (2000). Investigation of the relationship between movement development of 6-9 years old children and reasoning ability. Paper presented at the Marmara University 3. International Sports Sciences Congress Proceeding Booklet.

Izci, E., Kara, A., \& Dalaman, F. (2007). Investigation of classroom students in terms of multiple intelligence theory. Pamukkale University Journal of Education, 21, 1-14.

Kahraman, D. İ., \& Bavl, Ö. (2014). Investigation of multiple intelligence areas of students studying in different departments of the university. International Journal of New Trends in Arts, Sports and Science Education, 3(3).

Kahraman., S., \& Bulut, B. Ş. (2014). Investigation of multiple intelligence areas of gifted / gifted 6th, 7 th, 8 th grade students according to some variables. Journal of Gifted Education Research, 2(1), 58-73.

Katz, J., Mirenda, P., \& Auerbach, S. (2002). Instructional strategies and educational outcomes for students with developmental disabilities in inclusive "multiple intelligences" and typical inclusive classrooms. Research and Practice for Persons with Severe Disabilities, 27(4), 227-238.Available at: https://doi.org/10.2511/rpsd.27.4.227.

Kimura, D. (2002). Sex differences in the brain (revision of 1999 paper). Scientific American, 12, 32-37.

Kiremitçi, O., \& Canpolat, A. M. (2014). The role of physical education and sports school students in determining metacognitive awareness and problem solving skills of multiple intelligence areas. Hacettepe Journal of Sport Sciences, 25(3), 118--126.

Kul, M., Bozkuş, T., Erol, Z., \& Elçi, G. (2014). A study on the comparison of multiple intelligence areas of candidates eligible to enroll in physical education and sports school exams and candidates who fail to qualify. International Journal of Science Culture and Sport.

Kuru, E. (2001). Kinesthetic intelligence and physical education. Gazi Education Faculty Journal, 21(2), $217-229$.

Loori, A. A. (2005). Multiple intelligences: A comparative study between the preferences of males and females. Social Behavior and Personality: An International Journal, 33(1), 77-88.Available at: https://doi.org/10.2224/sbp.2005.33.1.77.

Mayer, J. D., Caruso, D. R., \& Salovey, P. (1999). Emotional intelligence meets traditional standards for an intelligence. Intelligence, 27(4), 267-298.Available at: https://doi.org/10.1016/s0160-2896(99)00016-1.

McClellan, J. A., \& Conti, G. J. (2008). Identifying the multiple intelligences of your students. Journal of Adult Education, 37(1), 13-32.

Metan, H., \& Küçük, V. (2017). Age group comparing individuals with and without regular sports with multiple intelligence aspects. Journal of International Social Research, 10(49), 327-333.

Moran, S., Kornhaber, M., \& Gardner, H. (2006). Orchestrating multiple intelligences. Educational Leadership, 64(1), 22-27.

Neville, A. L. (2000). Native American student's self perceptions regarding gardner's multiple intelligences. Doctor Theses, South Dakota State University, 51.

Nolen, J. L. (2003). Multiple intelligences in the classroom. Education, 124(1), 115-119.

Nulhakım, L., Wibawa, B., \& Erwın, T. N. (2019). Relationship between students' multiple intelligence-based instructional areas and assessment on academic achievements. Journal of Physics: Confernce Series.

Oral, B. (2001). Examining the intelligence areas of University students according to their branches. Education and Science, 26(122), 19-31.

Orhan, R. (2019). The importance of pysical activity and sport in child development. Krrlkkale University Journal of Social Sciences, 9(1), 157-

176.
Oztürkmen, B. (2006). Investigation of the relationship between secondary education students' intelligence areas and learning strategies according to multiple intelligence theory (The Case of Gaziantep). Unpublished Master Thesis. Gaziantep: Gaziantep University, Institute of Social Sciences.

Senel, E., \& Yıldız, M. (2016). The investigation of bodily/kinesthetic intelligence and sportspersonship orientation of students in school of physical education and sport. SSTB, 19, 54-61.

Teele, S. (1997). Teele inventory of multiple intelligences. Redlands, California: Sue Teele and Associates.

Tekin, M. (2007). Investigation of multiple intelligence areas of pre-service teachers studying in physical education and sports schools according to various variables. Paper presented at the 5 th Physical Education and Sports Teaching Symposium, Adana.

Tekin, M. (2008). Investigation of creativity and multiple intelligence areas among students who do and do not do sports in secondary education. Ph.D. Thesis, Gazi University, Institute of Educational Sciences, Ankara.

Tekin, M., Filiz, K., Tasgin, Ö., \& Özmutlu, İ. (2008). Comparison of multiple intelligence areas of students doing sports and non-doing sports in secondary education. Journal of Social Sciences Institute, 2, 1-10.

Temız, N., \& Kiraz, E. (2007). The implications of multiple intelligences theory on literacy education at first grade. Eurasian Journal of Educational Research, 27, 111-126.

Tunç, E. (2008). The relationship between multiple intelligence areas owned by 2nd year students and high school type area and gender. KEFD, 17, 108-130.

Ürgüp, S. (2015). Investigation of multiple intelligence areas of students studying in three different departments of school of physical education and sports. Master Thesis. Cumhuriyet University Institute of Health Sciences, Sivas.

Uysal, E. (2006). Investigation of children in the six-age group attending different preschool education institutions according to multiple intelligence theory. Master Thesis. Ankara University, Institute of Science. Ankara.

Yenice, N., \& Aktamış, H. (2010). Examining multiple intelligence areas of primary school teacher candidates according to demographic features. Journal of Turkish Science Education, 7(3), 86-99.

Yenilmez, K., \& Çalışkan, S. (2011). The relationship between primary education students' multiple intelligence areas and creative thinking levels. Dicle University Journal of Ziya Gökalp Education Faculty(17), 48-63.

Yıldız, M. (2010). Investigation of the relationship between active chess athletes studying in the 2nd stage of primary education and creativity and multiple intelligence areas. Unpublished Master Thesis. Gazi University, Ankara.

Zakowski, S. G., Harris, C., Krueger, N., Laubmeier, K. K., Garrett, S., Flanigan, R., \& Johnson, P. (2003). Social barriers to emotional expression and their relations to distress in male and female cancer patients. British Journal of Health Psychology, 8(3), 271286.Available at: https://doi.org/10.1348/135910703322370851.

Zimmer, R. (2014). Handbook movement education. Basics for training and educational practice. 1 st edition of the revised and expanded new edition 2013 (3rd ed.). Freiburg İm Breisgau: Verlag Herde. 